plugging. At 12 months post-discharge two (17\%) patients were dead; seven $(58 \%)$ were not on any ventilatory support; three $(25 \%)$ were continuing with tracheostomy ventilation.

Conclusion Respiratory weaning from tracheostomy ventilation represent a heterogenous group which is complex with diverse aetiology and multiple comorbidities. There is a considerable variation in the LOS on ARCU and is often unpredictable. Although more than two third of patients wean successfully on our unit it carries a high one year mortality. LOS is influenced by the complexity of discharge planning. We are not a dedicated weaning unit and our unit is not staffed to look after more than two tracheostomy-ventilated patients at any one time which combined with prolonged stay slows down patient flow from ICU to ARCU and from ARCU to the wards. Multidisciplinary approach and dedicated weaning units are needed that is able to look after complex needs in hospital and coordinate complex discharges.

\begin{tabular}{ll} 
Abstract P131 Table 1 & \\
\hline Age (mean+l-SD, years) & $56+/-17$ \\
\hline Males & $n=8$ \\
Females & $n=4$ \\
LOS in ICU pre tracheostomy & Median=8, Range=2-22 \\
& Mean- 9+/-6 \\
LOS in ICU post tracheostomy & Median=34, Range=7-96 \\
& Mean 41+/-30 \\
LOS in RCU post tracheostomy & Median=7, Range=1-93 \\
Primary Diagnosis (n=12) & Mean 21+/-33 \\
Pneumonia & \\
Post-procedure/surgery & 6 \\
COPD & 3 \\
ARDS & 1 \\
Other (Cardio-respiratory arrest) & 1 \\
\hline
\end{tabular}

\section{P132 THE ROLE OF VENTILATION IN PNEUMONIC EXACERBATIONS OF COPD}

${ }^{1}$ TM Hartley, ${ }^{1}$ ND Lane, ${ }^{1} \mathrm{~J}$ Steer, ${ }^{2} \mathrm{C}$ Echevarria, ${ }^{1} \mathrm{SC}$ Bourke. ${ }^{1}$ Northumbria Healthcare NHS Foundation Trust, North Shields, UK; ${ }^{2}$ The Newcastle upon Tyne Hospitals NHS Foundation Trust, Newcastle upon Tyne, UK

\subsection{6/thoraxjnl-2017-210983.274}

Introduction In isolated pneumonia, most trials show that NIV does not improve outcome, and may delay more appropriate intubation. However in pneumonia complicating COPD with acidaemic respiratory failure (AHRF), an RCT showed NIV reduced the need for intubation and conferred a survival benefit at 2 months. ${ }^{1}$ UK NIV guidelines state NIV is not indicated in pneumonia; whether this was intended to apply when pneumonia complicates another condition associated with a favourable response to NIV is unclear and there is substantial variation in practice. In our institution, most patients with pneumonic exacerbation of COPD (pECOPD) and AHRF receive NIV; few decline ventilation or are immediately intubated.

Methods From a consecutive historical cohort of patients receiving assisted ventilation for spirometry confirmed ECOPD and AHRF, chest radiographs, electronic data and clinical notes were reviewed. The presence of consolidation was determined in the following hierarchy: attending consultant physician interpretation (to mimic reality); radiologist report; or researcher interpretation. Analysis performed using IBM SPSS; significance identified using student's t-test, Mann Whitney $U$ or chi-squared test for parametric, non-parametric and categorical data respectively.

Results Among patients surviving to discharge, 90 day and 6 month mortality was $12.8 \%$ and $20.3 \%$ respectively in those with consolidation, compared to $12.9 \%$ and $18.4 \%$ respectively in those without.

Discussion Compared to those without pneumonia, patients with pECOPD were older, had more comorbid illnesses, more severe acidaemia and greater functional limitation. In addition, AHRF was more likely to have developed after admission, despite initial medical therapies (an adverse prognostic marker). Unsurprisingly, in-hospital mortality was significantly higher in those with pECOPD, but approximately $2 / 3$ survive to hospital discharge and post-discharge outcomes between the two groups are comparable. Coexistent consolidation is a marker of adverse acute outcome and an indication for closer monitoring but should not preclude ventilation, especially when so few are considered eligible for intubation.

\section{REFERENCE}

1. Confalonieri M, Potena A, Carbone $G$, et al. Acute respiratory failure in patients with severe community-acquired pneumonia: A prospective randomised evaluation of noninvasive ventilation. Am J Respir Crit Care Med 1999;160:1585-91.

Abstract P132 Table 1 Population Descriptors and in-hospital mortality by presence or absence of complicating pneumonia.

\begin{tabular}{llll}
\hline & $\begin{array}{l}\text { Consolidation } \\
\text { present } \\
\mathbf{n}=231\end{array}$ & $\begin{array}{l}\text { No } \\
\text { Consolidation }\end{array}$ & $\begin{array}{l}\text { Value } \\
\mathbf{n}=258\end{array}$ \\
\hline Age & $75.4(9.2)$ & $70.4(10.2)$ & $<0.001$ \\
FEV1 (\% predicted) & $41.0 \%(17.8)$ & $35.2 \%(14.6)$ & 0.001 \\
Current Smoker & $45.5 \%$ & $55.7 \%$ & 0.273 \\
BMI & $24.9(7.3)$ & $24.3(7.2)$ & 0.312 \\
eMRCD & $5(4-5)$ & $5(4-5)$ & 0.14 \\
Proportion 5a or 5b & $131(56.7 \%)$ & $133(51.6 \%)$ & 0.276 \\
Proportion 5b & $54(23.4 \%)$ & $46(17.8 \%)$ & 0.145 \\
Charlson Index & $2(1-3)$ & $1(1-2)$ & 0.042 \\
For escalation to Invasive & $7532.5 \%$ & $102(39.5 \%)$ & 0.1 \\
ventilation & & & \\
Received IPPV (+/-NIV) & $13(5.6 \%)$ & $14(5.4 \%)$ & 0.922 \\
Acidaemic on 1 st ABG & $186(80.5 \%)$ & $235(91.1 \%)$ & 0.001 \\
pH at ventilation initiation & $7.23(0.08)$ & $7.25(0.09)$ & 0.09 \\
C02 at ventilation initiation & $10.2(2.7)$ & $10.7(2.9)$ & 0.06 \\
CRP at NIV & $76(27-184)$ & $31(10-74)$ & $<0.001$ \\
Peak Pressure (IPAP) & $19(17-20)$ & $20(18-22)$ & 0.464 \\
In Hospital Mortality & $83 / 231(35.9 \%)$ & $41 / 258(15.9 \%)$ & $<0.001$ \\
\hline & & &
\end{tabular}

\section{P133 ACUTE HYPERCAPNIC RESPIRATORY FAILURE; APPLICATION OF A NOVEL HUMAN FACTORS APPROACH TO IMPROVE RECOGNITION AND MANAGEMENT}

${ }^{1} \mathrm{HJ}$ Pick, ${ }^{1} \mathrm{P}$ Cull, ' $\mathrm{E}$ Mullaney, ${ }^{1} \mathrm{~S}$ Smith, ${ }^{2} \mathrm{~N}$ Taylor, ${ }^{1} \mathrm{G}$ Lowrey. ${ }^{1}$ Royal Derby Hospital, Derby, UK; ${ }^{2} \mathrm{Hu}$-Tech Human Factors Ergonomics, London, UK

10.1136/thoraxjnl-2017-210983.275 